\title{
Towards the quantification of water quantity and quality impacts of rainwater tanks in South East Queensland
}

\author{
$\underline{\text { E.H. Coultas }}^{\text {a }}$, S. Maheepala ${ }^{\text {a }}$ and F. Mirza ${ }^{\text {a }}$ \\ ${ }^{a}$ CSIRO Land and Water, Highett, Victoria, Australia \\ Email: esther.coultas(a,csiro.au)
}

\begin{abstract}
To help ensure the security of water supply to urban catchments, a popular policy choice is to promote the installation of domestic rainwater tanks. While yield is primarily considered, this policy also causes a change in urban-runoff stormwater volumes and consequentially, nutrient export.
\end{abstract}

Modelling tools are used to predict the yield, volumetric reliability, overflow volume, and nutrient export that the deployment of rainwater tanks will cause. These modelling tools commonly utilise an up-scaling approach to analyse the behaviour of multiple tanks, where the performance of a single tank with average characteristics is linearly scaled up to represent a larger cluster of tanks.

Previous research has shown that this up-scaling method significantly overestimates the yield and volumetric reliability (Mitchell et al. 2008), and underestimates the overflow volume of the cluster (Neumann et al. 2011). A sensitivity analysis of the parameters used to represent a rainwater tank (roof catchment areas, tank storage capacities, demand) was carried out, using on-hand data for Melbourne water demand, rainfall and maximum temperature (Neumann et al. 2011). It identified that the non linearity of the tank yield and overflow in relation to some of the model parameters means that the adoption of an "average" (i.e. spatially lumped) tank to represent the behaviour of the entire cluster is subject to significant errors.

This paper establishes that, accordingly, nutrient export loads are also underestimated, and describes a stochastic water balance and quality model to effectively quantify the overflow nutrient load from a cluster of rainwater tanks at the catchment-scale.

In 2008, to reduce mains water consumption the South East Queensland region made rainwater tanks mandatory in new detached dwellings. It is important to accurately estimate overflow nutrient loads discharged to stormwater to identify changes at the catchment scale caused by this wide implementation of rainwater tanks.

The purpose of this paper is to apply the method described in Neumann et al (2011) and Maheepala et al (2011) to South East Queensland data, avoiding the limitations of spatial lumping of the performance of an average rainwater tank. This study examines the impact on overflow loads and volumes, and potable water savings at a catchment scale of a simulated cluster of RWTs in the Brisbane region, and also analyses the model's sensitivity to geographic climate parameters. By using Brisbane water demand, rainfall, and maximum temperature data, this paper illustrates the effect that the varied climates of Melbourne and Brisbane have on the results of the model, via different rainfall and usage patterns.

The results of this study for the Brisbane Local Government Area indicate an overestimation of volumetric reliability and yield, and an underestimation of the overflow volume and inflow load as well as the outflow load, which varies between $15 \%$ and $27 \%$, depending on the nutrient. Therefore it is not recommended to use an average tank to predict the performance of a cluster of household rainwater tanks to find their contribution to potable water savings, overflow volumes and subsequent nutrient and sediment export to stormwater. Instead, we recommend using stochastic simulation of rainwater tanks, which will include the use of probability distributions to represent tank characteristics and stochastic representation of end use water demands, calibrated using local climate and observed demand and rainwater tank data.

Keywords: $\quad$ Rainwater tank, Modelling, Nutrient export, Potable water savings, SEQ 
Coultas, Maheepala, Towards the quantification of water quantity and quality impacts of rainwater tanks in South East Queensland

\section{INTRODUCTION}

Domestic rainwater tank (RWT) systems are a popular option to alleviate the demand on potable water supply. The benefits of household rainwater usage also include the reduction of downstream storm water peak flows, and pollutant loads, particularly nitrogen (Mitchell 2007). When a policy decision is implemented at a city or a regional scale, such as in the South East Queensland region where rainwater tanks are mandatory in new dwellings and most new industrial and commercial buildings (Queensland Water Commission, 2010), it is vital to be able to assess the impact of rainwater tanks on potable water savings at a city and/or a regional scale and runoff at a catchment scale. The common method of assessing the impact at the catchment scale involves modelling an 'average' household RWT system, and scaling the results linearly to represent a larger cluster of households. Several researchers (Maheepala et al. 2011 and Mashford et al. 2011) showed that this method overestimates the average annual yield of a cluster of household rainwater tanks by about $18 \%$ for Canberra-based data and by about $14 \%$ for Melbourne based data. Mitchell et al. (2008) and Xu et al. (2010) also reported a similar magnitude of error for Melbourne-based data. In addition, Neumann et al (2011) examined the impact of ignoring the spatial variability of rainwater tank supplies on overflows from rainwater tank systems and subsequent nutrient discharges at catchment scale. They reported about $37 \%$ underestimation of catchment runoff and nutrient discharges using Melbourne data due to linear scaling up of performance of a single rainwater tank.

If an inaccurate modelling method is used to justify a city or regional-scale implementation of RWT systems, in reality the implementation policy is unlikely to reach targets for the reduction of potable demand and nutrient discharge.

The purpose of this paper is to apply the method described in Neumann et al (2011) and Maheepala et al (2011) to South East Queensland data, avoiding the limitations of spatial lumping of the performance of an average rainwater tank (i.e. linear scale-up method). This is done by stochastic simulation of a cluster of household rainwater tanks using the rainwater tank model reported in Mitchell et al. (2008), hereafter referred to as the RWT model.

As part of the study, the RWT model has been enhanced to simulate in-tank routing and water quality, in order to simulate nutrient and sediment overflow loads, as well as overflow volume. Users of the RWT model can still stochastically simulate many households by defining distributions for household parameters, and now also benefit from more accurate modelling of the water quality within the RWT and therefore of discharged loads. These simulated-households make up a more accurate representation of a large cluster of households spread across a catchment, a city or a region.

This study examines the impact on overflow loads and volumes, and potable water savings at a catchment scale of a simulated cluster of RWTs in the Brisbane region, using the enhanced RWT model and a Stochastic Demand Generator model (Duncan and Mitchell, 2008) for toilet and clothes washer demand, calibrated to observed household end use data from Brisbane Local Government Area (Beal et al. 2010) for one and two person households. The results of this stochastic simulation are then compared to the common method of up-scaling an 'average' house. The study will continue for the next few months to calibrate the Stochastic Demand Generator model (Duncan and Mitchell, 2008) to other end uses in Brisbane Local Government Area (LGA) as well as Ipswich LGA, Sunshine Coast LGA and Gold Coast LGA.

\subsection{Brisbane Case Study Area}

In view of the South East Queensland region mandate that new dwellings must implement rainwater tanks (Queensland Water Commission, 2010), we study the Brisbane area to calculate the contribution these new RWTs will make to overflow loads of sediment / nutrient discharge to stormwater. This paper reports the impact of having rainwater tanks in one and two person households only. The work is in progress to include other plausible occupancy rates in the South East Queensland. As per (ABS 2006), the ratio between one to two person households in Brisbane is 22:34.

The total simulation period was 50 years, from 1960 to 2010 . The rainfall and evaporation time series were obtained from the Bureau of Meteorology Brisbane Regional Office (station 40214). During the period 1960 to 2010 , the mean annual rainfall was $1093 \mathrm{~mm} /$ year while the mean annual areal potential evaporation was $1605 \mathrm{~mm} /$ year.

\section{METHOD}

To evaluate the over or under estimation of the rainwater tank overflow load caused by linear up-scaling of the performance of an average RWT, our method involved simulating the behaviour of a 5000 house cluster 
Coultas, Maheepala, Towards the quantification of water quantity and quality impacts of rainwater tanks in South East Queensland

of rainwater tank systems with varied parameters (roof area, tank size, initial and continuing losses, occupancy), hereafter referred as the variable case. The simulation of the performance of RWT cluster was carried out using the RWT model and plausible patterns of demand for toilet and clothes washers were generated using the Stochastic Demand Generator model. The variable case was compared with a second scenario that involved linear up-scaling of tanks with averaged values, hereafter referred as the mean case. The averaged parameters are computed from the distribution of parameters used in the variable case using arithmetic means.

A number of measures were used to compare the performance of "variable" and "mean" cases. The measures were: average annual rainwater tank yield (i.e. the volume of water supplied by rainwater tanks on an average annual basis over the simulation period), volumetric reliability (i.e. the total volume of water supplied by RWTs divided by the total target demand volume), average annual overflow volume (i.e. the overflow volume of water from rainwater tanks on an average annual basis over the simulation period) and average annual total nitrogen (TN), total prosperous (TP) and total suspended solids (TSS) loads (i.e. the TN, TP and TSS loads on an average annual basis over the simulation period).

\subsection{Modelled Scenarios}

As mentioned earlier, two scenarios (or cases) were considered for the Brisbane-based data. They are named as Brisbane variable case and Brisbane mean case, to evaluate the affect of linear up-scaling on water quantity and quality performance of a rainwater tank cluster. In addition, results of the variable case of Melbourne-based data (obtained from Maheepala et al. 2011 and Neumann et al. 2011) are presented in this paper for comparison purposes. All these cases are described in Table 1.

Table 1 RWT Model input parameters

Note: $\mathrm{ND}^{1}=$ Normal Distribution as per Table 2. $\mathrm{SGD}^{2}=$ Stochastic Demand Generator

\begin{tabular}{|l|l|l|l|l|l|}
\hline Description & Tank & Roof & $\begin{array}{l}\text { Initial } \\
\text { loss }\end{array}$ & $\begin{array}{l}\text { Continuing } \\
\text { loss }\end{array}$ & Demand \\
\hline Brisbane Variable case & $\mathrm{ND}^{1}$ & $\mathrm{ND}^{1}$ & $\mathrm{ND}^{1}$ & $\mathrm{ND}^{1}$ & 100 profiles generated using the SDG \\
\hline Brisbane mean (arithmetic) case & 2.6 & 106.8 & 0.634 & 14.9 & Mean demand profile generated \\
\hline Melbourne Variable case & $\mathrm{ND}^{1}$ & $\mathrm{ND}^{1}$ & $\mathrm{ND}^{1}$ & $\mathrm{ND}^{1}$ & 100 profiles generated using the $\mathrm{SDG}^{2}$ \\
\hline
\end{tabular}

\subsection{Simulation of water quantity and quality of a cluster of household rainwater tanks}

To simulate both water quantity and quality of a cluster of household rainwater tanks, the RWT model of Mitchell et al (2008) was modified to include nutrients and sediments. The original RWT model uses two modules to perform a continuous simulation: a rainfall runoff module to represent the roof and a storage model to represent the rainwater tank, as shown in Figure 1.The rainfall runoff module requires two time series as inputs: a rainfall time series, and a potential evaporation time series. It also has three parameters, the roof area, an initial loss which represent the depression storage, and a continuing loss which represents continuous losses due to splashing, gutter overflow, effectively reducing the roof runoff by a loss factor. The storage model uses a simple water balance to represent the rainwater tank, and it contains two inputs, the inflow from the roof and a demand time series, and one parameter, the tank volume. Evaporation from the tank and leakage losses are neglected by assuming the tank is closed and is leak-free. The model has the capability to represent requested demand (from the tank) for multiple end-uses. Besides rainfall, potential evaporation and demand, these parameters can be specified as either a single value or a continuous probability distribution. For a complete description of the model, the user is referred to Mitchell et al (2008).

To improve nutrient export simulation, we implemented Puls method (Institution of Engineers Australia, 2001) in-tank hydrologic routing in the RWT model. This algorithm models the behaviour of rainfall run-off as it flows through the rainwater tank. We further enhanced the model to include an implementation of the first order kinetic decay model (the "k-C" model) based on the "Continuous Stirred Tank Reactor" (CSTR) concept as described by Wong (2006). In addition to the k-c model, a stochastic pollutant generation algorithm was also implemented. The pollutant generation follows the method described in the MUSIC software v3 (CRC-CH 2005), where there is no correlation between the stochastic generated values between two subsequent steps. 
Coultas, Maheepala, Towards the quantification of water quantity and quality impacts of rainwater tanks in South East Queensland

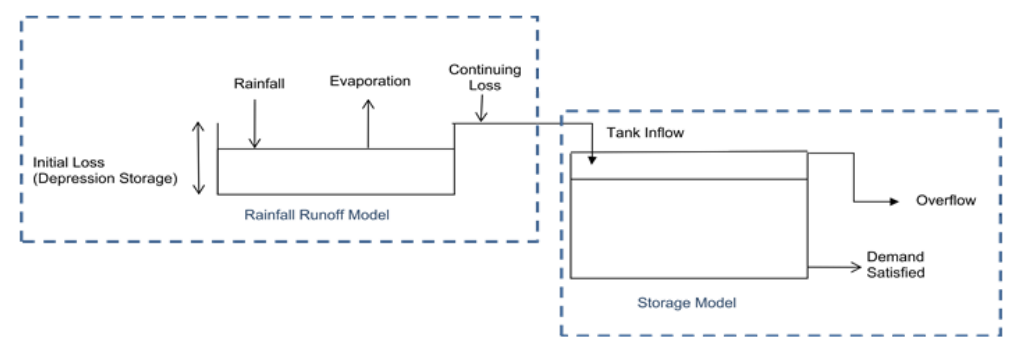

Figure 1 Schematic representation of the rainwater tank model (source: Neumann 2011, adapted from Mitchell et al. (2008).

\section{RWT Model input parameters}

The variable case draws parameter values from the normal distributions specified in Table 2 to enable it to stochastically model a cluster of 5000 separate households. These parameters represent typical values (sourced from Mitchell et al. 2008) and will be modified by using the values relevant to South East Queensland as the study progresses towards to a full application in the South East Queensland. The arithmetic mean case models a single house using average values derived from running a large cluster of variable houses, the simulated results of which are then linearly up-scaled by multiplying by 5000 .

Table 2 Assumed normal distribution parameters and maximum and minimum values used to generate the variable case

\begin{tabular}{|l|l|l|l|l|l|}
\hline Parameter & Min & Mean & Max & Standard Deviation & Rounding (i.e. precision) \\
\hline Tank capacity $(\mathrm{kL})$ & 0.1 & 2.5 & 20 & 1.5 & 0.1 \\
\hline Roof area $\left(\mathrm{m}^{2}\right)$ & 25 & 100 & 400 & 50 & 5 \\
\hline Initial Loss $(\mathrm{mm})$ & 0 & 0.5 & 1.7 & 0.5 & 0.01 \\
\hline Continuing loss $(\%)$ & 0 & 15 & 30 & 5 & 0.05 \\
\hline
\end{tabular}

To perform a geographical comparison, the Melbourne variable case uses the same variable case model parameters found in Neumann et al (2011), but only for one and two person households and for Toilet and Clothes washer end-uses in order to be comparable with the available Brisbane data. For details of parameters of the Melbourne variable case, see Maheepala et al (2011) and Neumann et al (2011). For the water quality simulation, the values used for $\mathrm{k}$ and $\mathrm{C}^{*}$ are the default MUSIC model values for each of TSS, TN and TP (CRC-CH 2005). The Concentration inflow rate $(\mathrm{mg} / \mathrm{l})$ uses a log-normal distribution with a minimum of 1 , a mean of 20 , maximum of 200 , rounding of 1 , and standard deviation of 47 .

\subsection{Generation of end use demand}

As mentioned earlier, the Stochastic Demand Generator of Duncan and Mitchell (2008) was used to generate demand time series of end uses that are representative for Brisbane. The model was calibrated to Brisbane for one and two person households.

\section{Stochastic Demand Generator (SDG) Model Input Parameters}

Input data for the SDG model (Duncan and Mitchell, 2008) are shown in Table 3. However, at the time of undertaking the study, the format of the measured data available for each end use in Brisbane LGA was in litres/person/day (or 1/p/d) (Beal et al, 2010). Hence, we considered the variables shown in Table 4 as parameters of the SDG. Calibration of the SDG model involved calibration of these parameters to obtain simulated end use values as close as possible to the observed end use values. The SDG model uses a oneminute time step, which is then aggregated into time series' of 6-minute, hourly and daily time steps.

This paper assumed that rainwater was used for toilet and clothes washer usage only. This study used observed data of Brisbane LGA during a two-week period in winter 2010 (June 2010) (Beal et al. 2010). The observed data sampled 61 homes in Brisbane LGA. The household size of the sample varied from 1 to 6 . We used only 1 and 2 household sizes for the preliminary analysis reported in this paper. The mean and standard deviations used for the calibration are shown in Table 4. The resulting calibration parameters are shown in Table 5. This study reports preliminary results that were based on Brisbane data only. Analysis will be conducted by using the inputs data shown in Table 3 and for the other LGAs (i.e. Ipswich, Gold Coast and Sunshine Coast) and all occupancy rates in the future studies in order to represent household water demand of the whole SEQ. 
Coultas, Maheepala, Towards the quantification of water quantity and quality impacts of rainwater tanks in South East Queensland

Table 3 Input data of the Stochastic Demand Generator Model

\begin{tabular}{|l|l|l|l|}
\hline \multirow{2}{*}{3.} & \multicolumn{2}{|l|}{ Input data } \\
\hline End use & Frequency & Volume in litres per event & Other \\
\hline Toilet & No of half flushes/day & Half flush volume for each flush & \\
\cline { 2 - 4 } & No of full flushes/day & full flush volume for each flush & \\
\hline Taps and hand basins & No of uses per day & volume per use & \\
\hline Showers & No of showers/day & $\begin{array}{l}\text { duration per event and shower } \\
\text { flow rate in litres/min }\end{array}$ & \\
\hline Bath & No of baths/day & Bath volume and filling rate & \\
\hline Dishwashers & No of washes/day & volume per wash & Presence of a dishwasher \\
\hline Clothes washers & No of washes/day & volume per wash & $\begin{array}{l}\text { Presence of a clothes washer and the type (i.e. } \\
\text { Type: Top Loader or Front Loader) }\end{array}$ \\
\hline Garden watering & No of events & $\begin{array}{l}\text { flow rate in litres/min and } \\
\text { duration per event }\end{array}$ & $\begin{array}{l}\text { A function that specifies the occurrence of } \\
\text { garden watering in any time step of a given } \\
\text { day as a function of climatic variables such as } \\
\text { maximum temperature, evaporation and/or } \\
\text { rainfall }\end{array}$ \\
\hline
\end{tabular}

Table 4 Summary of observed Brisbane end-use data for 1 and 2 person-households (phh) (source: Beal et al, 2010)

\begin{tabular}{|c|c|c|c|c|c|c|}
\hline & $\begin{array}{l}\text { Toilet } \\
\text { (1/p/d) }\end{array}$ & $\begin{array}{r}\text { Clothes Washer } \\
(\mathbf{l} / \mathbf{p} / \mathbf{d})\end{array}$ & $\begin{array}{r}\text { Shower } \\
(1 / p / d)\end{array}$ & $\begin{array}{r}\text { Dishwasher } \\
(\mathbf{l} / \mathbf{p} / \mathbf{d})\end{array}$ & $\begin{array}{r}\text { Tap } \\
(\mathbf{l} / \mathbf{p} / \mathbf{d})\end{array}$ & $\begin{array}{r}\text { Bathtub } \\
(\mathrm{l} / \mathrm{p} / \mathrm{d})\end{array}$ \\
\hline 1 phh Observed Mean & 31.9467 & 33.7534 & 43.0669 & 1.3917 & 33.2205 & 0 \\
\hline 1 phh Observed SD & 9.9753 & 15.1597 & 17.9108 & 2.5792 & 13.1305 & 0 \\
\hline 2 phh Observed Mean & 25.7855 & 38.1719 & 40.9337 & 1.7105 & 24.6791 & 0.8618 \\
\hline 2 phh Observed SD & 13.1329 & 20.4059 & 19.8465 & 2.4584 & 10.4843 & 2.5229 \\
\hline
\end{tabular}

Normality of the observed data of 1 and 2 person households was tested using chi-square goodness of fit test, which indicated log-normal distribution to toilets and normal distribution to all other end uses. Accordingly, the SDG was modified in the following ways: Shower was changed from log-normal to normal distribution, toilet was changed from normal to lognormal distribution, taps were changed from a fixed value to a normal distribution, and clothes washer was changed from fixed distribution to normal distribution. Dishwasher uses a fixed value for volume, and bath uses a fixed volume and fixed flow rate - these did not need to be modified. The SDG model was calibrated to 1 and 2 person

Table 5 Brisbane observed and modelled average demand

\begin{tabular}{|l|r|r|}
\hline Household occupancy: & \multicolumn{1}{|c|}{ One } & \multicolumn{1}{c|}{ Two } \\
\hline Average observed toilet demand (1/p/d) & 31.95 & 25.78 \\
\hline Average modelled (using SDG) toilet demand (1/p/d) & 30.34 & 25.36 \\
\hline Average observed clothes washer demand (1/p/d) & 33.75 & 38.17 \\
\hline Average modelled clothes washer demand (1/p/d) & 33.46 & 38.50 \\
\hline
\end{tabular}
household sizes (or occupancy rates) and toilet and clothes washer uses. Results (i.e. observed and modelled water demands of toilet and clothes washer end uses for 1 and 2 person households) are shown in Table 5.

The calibrated SDG model was then used to generate representative end use demand time series in order to simulate the performance of 5000 household rainwater tanks. One hundred demand time series were generated. Of the hundred time series, 39 represented one-person households and 61 represented two-person households, which maintained 22:34 ratio as per (ABS 2006).

\section{Mean case demand input parameters}

The demand time series were averaged on a time step basis. The values for each time step across all 100 demand files averaged using the arithmetic mean to generate the time series for the mean case. This demand time series represents the arithmetic mean of all demand.

\section{RESULTS AND DISCUSSION}

Differences between the mean case (i.e. with spatial lumping) and variable case (i.e. without spatial lumping) for the different performance measures considered in this study are shown in Table 6. It shows that spatial lumping of rainwater tanks overestimates potable water savings by about $15 \%$ compared to a case without spatial lumping. This result is comparable with $18 \%$ overestimation reported for Canberra-based data 
Coultas, Maheepala, Towards the quantification of water quantity and quality impacts of rainwater tanks in South East Queensland

(Maheepala et al. 2011) and 14\% overestimation reported for Melbourne-based data (Xu et al. 2010 and Mitchell et al. 2008). However, it should be noted that the results based on Brisbane-based data assumed rainwater for toilet and clothes washer uses only. If rainwater is used for garden water use, the variable case will incorporate more spatial variability than the spatial variability present in the results, which may likely to result in more overestimation than $15 \%$.

Table 6 Comparison of performance of a cluster of household rainwater tanks with and without the spatial lumping effect

\begin{tabular}{|l|c|c|c|}
\hline & $\begin{array}{l}\text { Mean case } \\
\text { spatial lumping effect) }\end{array}$ & $\begin{array}{l}\text { Variable case (without spatial } \\
\text { lumping effect) }\end{array}$ & $\begin{array}{l}\text { Difference between mean and } \\
\text { variable cases as a percent of } \\
\text { mean case }\end{array}$ \\
\hline Volumetric reliability & 0.82 & 0.70 & $-14.7 \%$ \\
\hline Tank yield (kL/year) & 30.86 & 26.30 & $-14.8 \%$ \\
\hline Overflow volume (kL/year) & 64.26 & 68.28 & $6.3 \%$ \\
\hline TSS/TN/TP Inflow load (kg/year) & 2.23 & 2.36 & $6.0 \%$ \\
\hline TSS Overflow load (kg/year) & $\mathbf{1 . 0 9}$ & $\mathbf{1 . 2 9}$ & $\mathbf{1 7 . 8 \%}$ \\
\hline TN Overflow load (kg/year) & $\mathbf{1 . 2 0}$ & $\mathbf{1 . 3 9}$ & $\mathbf{1 5 . 5 \%}$ \\
\hline TP Overflow load (kg/year) & $\mathbf{0 . 7 7}$ & $\mathbf{0 . 9 8}$ & $\mathbf{2 7 . 6 \%}$ \\
\hline
\end{tabular}

The calculated results from running the mean and variable case for each of the three pollutants: total suspended solids (TSS), total nitrogen (TN) and total phosphorous (TP), are shown in Table 6. The results indicate an underestimation of the overflow volume and inflow load as well as the outflow loads, which varies between $15 \%$ and $27 \%$, depending on the nutrient: $17.8 \%$ for TSS, $15.5 \%$ for TN. Table 7 contains the results from simulating the comparatively more 'tropical' Brisbane variable case and also a comparable case, using 'temperate' Melbourne demand, rainfall and evaporative data.

Table 7 Comparison of performance of a cluster of household rainwater tanks without the spatial lumping effect for Brisbane and Melbourne data

\begin{tabular}{|l|l|l|}
\hline & Brisbane variable case & Melbourne variable case \\
\hline Volumetric reliability & 0.70 & 0.63 \\
\hline Demand requested (kL/year) & 37.36 & 52.31 \\
\hline Tank yield (kL/year) & 26.30 & 32.79 \\
\hline Overflow volume (kL/year) & 68.28 & 18.89 \\
\hline TSS Inflow load (kg/year) & 2.36 & 1.29 \\
\hline TSS Overflow load (kg/year) & 1.29 & 0.33 \\
\hline TSS change (kg/year) & -3.65 & -1.62 \\
\hline TSS reduction (\%) & $-45 \%$ & -0.74 \\
\hline
\end{tabular}

The geographical comparison showed that the results are similar, apart from overflow load. Due to the higher rainfall and lower demand in Brisbane has an overflow volume and load almost three times higher than Melbourne. This demonstrates sensitivity of results to local climatic and water use data, and illustrates the importance of calibrating the Stochastic Demand Generator and RWT models with local climate and usage characteristics from the catchment area of interest.

\section{CONCLUSIONS AND RECOMMENDATIONS}

In this study we have investigated the effect of spatial lumping of household rainwater tank supply on potable water savings, overflow volumes and nutrient and sediment loads, and also briefly compared the results of similar scenarios in different geographical areas: tropical Brisbane and temperate Melbourne. Results of this study indicates that ignoring of the spatial variability of water use from rainwater tanks and rainwater tank characteristics can cause overestimation of potable water savings and underestimation of overflow volumes as well as the nutrient and sediment loads associated with the overflow volumes, obtained from a cluster of rainwater tanks.

Results reported in this study should be considered as "indicative" because of the assumptions made with regard to rainwater tank parameters values, limited use of rainwater for toilet and clothes washer uses and the 
Coultas, Maheepala, Towards the quantification of water quantity and quality impacts of rainwater tanks in South East Queensland

limited availability of observed end use data relevant to South East Queensland. Nevertheless, the preliminary results are of similar magnitude to the results reported previous research studies such as Mitchell et al (2008), Maheepala et al. (2011), Mashford et al. (2011) and Neumann et al (2011). Comparison of Melbourne based results with those of Brisbane has indicated that the effect of spatial lumping can vary with the local climate.

The key outcome of the study thus far is that it is not recommended to use an average tank to predict the performance of a cluster of household rainwater tanks to find their contribution to potable water savings, overflow volumes and subsequent nutrient and sediment export to stormwater. Instead, we recommend using stochastic simulation of rainwater tanks, which will include the use of probability distributions to represent tank characteristics and stochastic representation of end use water demands, calibrated using local climate and observed demand and rainwater tank data.

\subsection{Further Work}

Analysis will be conducted for all council areas in SEQ in future studies to examine the impact of rainwater tanks on SEQ's supply/demand balance. Observed Brisbane garden watering end-use data shows a close correlation with daily maximum temperature. We will be examining the validity of this relationship in our future work, with a view to stochastically generating suitable garden end-use demand time series'. Acquisition of observed demand data for households of all occupancies for all SEQ council areas will enable us to calibrate the demand model for all occupancies.

\section{ACKNOWLEDGMENTS}

This research was funded by the South East Queensland Urban Water Security Research Alliance, a scientific collaboration between the Queensland Government, CSIRO, The University of Queensland and Griffith University. The authors would like to thank Hugh Duncan for providing access to the demand generator.

\section{REFERENCES}

ABS. 2006. "Brisbane (Statistical Division), 40214 - Household Composition by Number of Persons Usually Resident - Brisbane."

Beal, C.D., Stewart, R.A., Huang, T. 2010. South East Queensland Residential End Use Study: Baseline results - Winter 2010. Urban Water Security Research Alliance Technical Report No. 31.

CRC-CH, 2005. Model for Urban Stormwater Improvement Conceptualisation (Music) (Version 3). Melbourne, Australia, Cooperative Research Centre for Catchment Hydrology.

Duncan, H. P. and Mitchell, V., 2008. A Stochastic Demand Generator for Domestic Water Use. Proceedings of Water Down Under 2008, Modbury, SA: Engineers Australia, Causal Productions.

Maheepala, S., Loonat, N, Mirza, F., and Coultas, E., 2011. Quantifying potable water savings of rainwater tanks at a city scale by considering the effect of spatial lumping, OZWater 2011, 09-11 May 2011, Adelaide, Australia.

Mashford, J., Maheepala, S., Neumann, L., and Coultas, E.H., 2011. Computation of the expected value and variance of the average annual yield for a stochastic simulation of rainwater tank clusters, In press.

Mitchell, V., Siriwardene, N., Duncan, H. and Rahilly, M., 2008. Investigating the Impact of Temporal and Spatial Lumping on Rainwater Tank System Modelling. International Conference on Water Resources and Environment Research, Modbury, SA, Engineers Australia ; Causal Productions.

Mitchell, V. G., 2007. How Important Is the Selection of Computational Analysis Method to the Accuracy of Rainwater Tank Behaviour Modelling? Hydrological processes 21(21): 2850-2861.

Neumann L.E., Coultas. E.H., Moglia M., and Mashford J.. in press. Errors in yield and overflow estimation in rainwater tank cluster modelling. In press conference proceedings of 12th International Conference on Urban Drainage, Porto Alegre/Brazil, 11-16 September 2011.

Wong, T. H. F., Fletcher, T. D., Duncan, H. P. and Jenkins, G. A., 2006. Modelling Urban Stormwater Treatment--a Unified Approach. Ecological Engineering 27(1): 58-70.

Xu, H., Rahilly, M. and Maheepala, S., 2010. Assessing the Impact of Spatial Lumping on Rainwater Tank Performance Using Daily Modelling. 9th International Conference on Hydro-informatics, Chinese Academy of Sciences, China. 